\title{
Source analysis reveals plasticity in the auditory cortex: Evidence for reduced hemispheric asymmetries following unilateral deafness
}

\author{
Michael R.D. Maslin*, Kevin J. Munro, Wael El-Deredy \\ School of Psychological Sciences, University of Manchester, Oxford Road, Manchester M13 9PL, United Kingdom
}

\section{A R T I C L E I N F O}

\section{Article history:}

Accepted 31 July 2012

Available online $\mathrm{xxxx}$

\section{Keywords:}

Experience-related plasticity

Evoked potentials

Dipole source analysis

N1

Unilateral deafness

Hemispheric asymmetries

\begin{abstract}
H I G H L I G H T S
- Experience-related plasticity is apparent in adult humans with profound unilateral deafness, reflected by changes in dipole strength, location and orientation.

- Inconsistencies in previous studies using dipole source analysis may be explained by inadequate consideration of the effect of dipole location and orientation.

- This study provides evidence for experience-related plasticity in the adult central auditory system, and the results may help to clarify the meaning of altered dipole strengths, location and orientation parameters.
\end{abstract}

\begin{abstract}
A B S T R A C T
Objective: To investigate the effect of acquired unilateral deafness on hemispheric asymmetries in adult humans using cortical auditory evoked potentials.

Methods: N1 cortical auditory evoked potentials were measured from 30 channels in 18 unilateral profoundly-deaf participants ( 6 right-sided and 12 left-sided deafness) and 18 audiogram-matched controls. Stimuli were $0.5-\mathrm{kHz}$ and $4-\mathrm{kHz}$ tones presented monaurally, and the data were analysed using global field power and dipole source analysis.

Results: There was a statistically significant difference in dipole source strength and orientation between the two groups. Similar changes (increased dipole strength and more medial orientation) were apparent after profound unilateral deafness of either ear and for both stimuli.

Conclusions: The results reveal evidence of central auditory system plasticity that is consistent with animal models having experimentally induced unilateral deafness.

Significance: The trend towards reduced hemispheric asymmetries was reflected in the dipole source model by changes in dipole strength, location and orientation. These findings may explain the inconsistencies reported in previous studies involving dipole source analysis where location and orientation have not always been considered.

(c) 2012 International Federation of Clinical Neurophysiology. Published by Elsevier Ireland Ltd. All rights
\end{abstract} reserved.

\section{Introduction}

The central auditory system (CAS) is known to exhibit experience-related plasticity following the onset of deafness (Neuman, 2005). Evidence of auditory plasticity in mature humans has been obtained from studying individuals with unilateral deafness (Scheffler et al., 1998; Bilecen et al., 2000; Ponton et al., 2001; Langers et al., 2005; Firszt et al., 2006). In normal hearing individuals, monaural stimulation results in asymmetrical activation of the CAS, with the contralateral hemisphere producing greater activation than the ipsilateral hemisphere (Woldorff et al., 1999; Hine and Debener, 2007). This is referred to here as hemispheric

\footnotetext{
* Corresponding author. Tel.: +44 (0) 161275 3459; fax: +44 (0) 1612753373.

E-mail address: michael.maslin@manchester.ac.uk (M.R.D. Maslin).
}

asymmetry. Profound unilateral deafness leads to changes in the normal pattern of hemispheric asymmetry upon stimulation of the remaining intact ear. These changes consist primarily of an increase in the response of the hemisphere ipsilateral to the ear of stimulation, while activity in the contralateral hemisphere remains largely unchanged (Popelar et al., 1994; McAlpine et al., 1997). Therefore, monaural stimulation in individuals with unilateral deafness results in a greater overall response of the CAS and reduced hemispheric asymmetry. However, whilst data from functional magnetic resonance imaging (fMRI) studies have consistently shown these changes in humans with profound unilateral deafness (Scheffler et al., 1998; Bilecen et al., 2000; Langers et al., 2005; Firszt et al., 2006), this has not been the case in studies using magnetoencephalography (MEG) and electroencephalography (EEG). To date there has only been one published study, using 
cortical auditory evoked potentials (CAEPs), that has demonstrated experience-related plasticity in humans with late-onset profound unilateral deafness (Ponton et al., 2001). CAEPs produced using a click stimulus were measured from 15 individuals with unilateral deafness and compared with those of nine normal hearing controls. A key aspect of the methodology of this study was that the analysis was based on sensor level data, and was carried out in two stages. First, the root-mean-squared amplitude of CAEPs across groups of sensors, over each hemisphere, was compared. Second, the amplitude of the N1 obligatory CAEP across subsets of sensors was compared. Results from the first analysis showed that while the response amplitude over the contralateral hemisphere was similar between the groups of participants, the amplitude over the ipsilateral hemisphere was significantly greater in those with unilateral deafness, resulting in reduced hemispheric asymmetry. Furthermore, these changes were subsequently found to be reflected primarily by sensors in the central scalp regions. Despite this finding, most studies involving the N1 wave in CAEPs or its MEG equivalent, $\mathrm{N} 1 \mathrm{~m}$, have not consistently shown any evidence of plasticity following unilateral deafness in adult humans (Vasama et al., 1995, 2001; Fujiki et al., 1998; Khosla et al., 2003; Hine et al., 2008; Hanss et al., 2009). The present study was therefore conducted with the aim of clarifying the reasons for the inconsistent findings in EEG/MEG studies of plasticity in adult humans with late onset profound unilateral deafness.

One explanation for these inconsistencies could be methodological factors involving the source modeling techniques most often used to characterize the response of the CAS (Vasama et al., 1995, 2001; Fujiki et al., 1998; Khosla et al., 2003; Hine et al., 2008). CAEPs such as the $\mathrm{N} 1$ (or $\mathrm{N} 1 \mathrm{~m}$ ) are usually characterized in source models by one or more dipoles in each hemisphere. Measuring hemispheric asymmetries therefore becomes a case of comparing the strength, location and orientation of the dipoles in each hemisphere. In normal hearing individuals the contralateral dipole would be expected to have a greater strength than the ipsilateral dipole following monaural stimulation (Woldorff et al., 1999; Hine and Debener, 2007). Previous studies investigating changes in CAS activity following unilateral deafness have focused on changes in dipole strength, and have not always considered changes in orientation or location of the dipole (Vasama et al., 1995, 2001; Fujiki et al., 1998; Khosla et al., 2003; Hine et al., 2008). However, it is possible that changes in the normally observed asymmetrical CAS response may be reflected by changes in dipole strength, location, orientation or complex interactions between all three parameters in each hemisphere.

The present study therefore aimed to characterize any differences in N1 CAEPs between adults with profound unilateral deafness and a control group by measuring the degree of activation produced over each hemisphere in the N1 latency range to monaural stimulation. The analysis involved consideration of dipole strength, location and orientation since this has not been done in previous such studies. It was hypothesized that an overall increase in CAEP response amplitude would be apparent in those with unilateral deafness, reflecting an increased responsiveness of CAS neurons to stimulation of the intact ear, but that reduced hemispheric asymmetries may be reflected by changes in the relative dipole strengths, locations and orientations in each hemisphere.

\section{Methods}

\subsection{Participants}

Eighteen unilateral profoundly deaf adults (UDs) took part in the study ( 5 female, 13 male; mean age $=60$ years old, range $=43-75$ years old). Of these, 6 had right- and 12 had

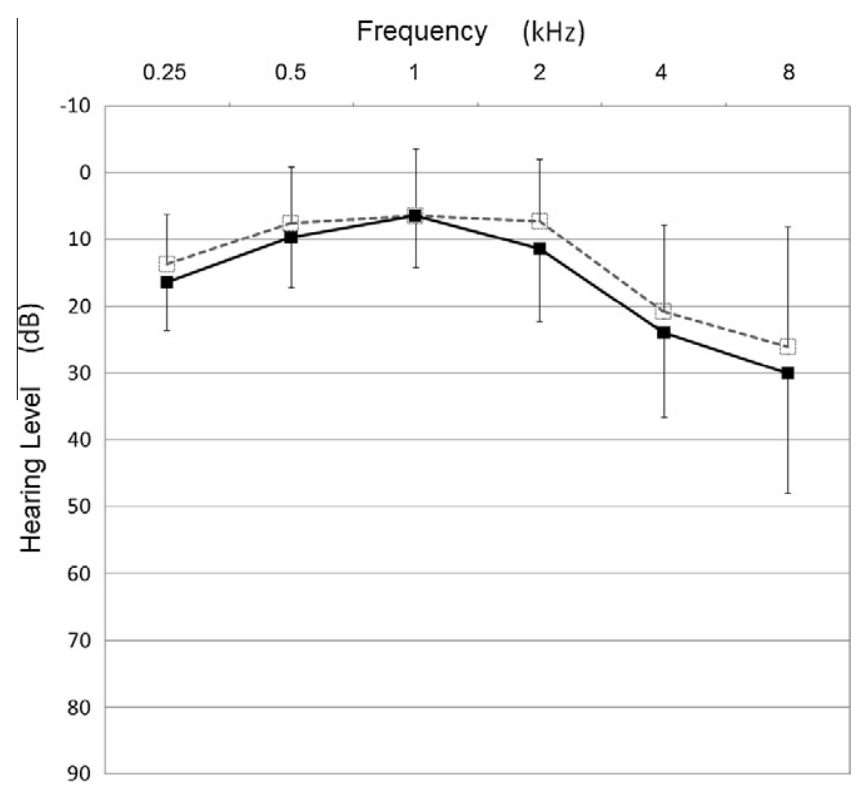

Fig. 1. Mean pure tone hearing thresholds for the test ear of unilaterally deaf (filled symbols) and matched control participants (open symbols). Error bars denote \pm 1 s.d.

left-sided deafness due to translabyrinthine surgery for the removal of a unilateral acoustic neuroma. This surgery results in abrupt and complete deafness of the affected side. The duration of deafness after surgery ranged from 6 months to 7 years and all participants were right handed according to the Edinburgh Handedness Inventory (Oldfield, 1971). Matched to each individual was a control participant (MCs) with hearing threshold levels in both ears similar to the participant's intact ear $( \pm 10 \mathrm{~dB}$ at 0.5 $\mathrm{kHz}$ and $4-\mathrm{kHz}$ ). The participants were also matched for sex, handedness and age (controls mean age $=58$ years old, range $=42$ 74 years old). The mean pure tone hearing threshold levels for participants and matched controls is shown in Fig. 1. The study was approved by the Cumbria and Lancashire NHS Research Ethics Committee (08/H1016/66) and all participants gave written, informed consent.

\subsection{Stimuli}

Two pure tones $(0.5-\mathrm{kHz}$ and $4-\mathrm{kHz})$ were selected to represent low and high-frequency stimuli. These were of $80 \mathrm{~ms}$ duration including $10 \mathrm{~ms}$ onset and offset ramps, defined using the Blackmann windowing algorithm. The stimuli were generated digitally and presented via a $44.1 \mathrm{kHz}$ digital-analogue converter monaurally to the intact ear of participants with unilateral deafness, and the corresponding ear in control participants. Stimuli were presented in a pseudo randomly interleaved fashion via ER-3A insert earphones. The inter-stimulus interval varied pseudo randomly between 900 and 1300 ms. Each stimulus was presented in blocks of 500 repetitions and the order of frequency of presentation was counterbalanced across participants. The presentation level was set at a sensation level (SL) of $60 \mathrm{~dB}$, although three participants and their matched controls received reduced SLs of $40 \mathrm{~dB}$ in the $4-\mathrm{kHz}$ condition due to subjective loudness discomfort. The absolute levels varied by up to $30 \mathrm{~dB}$ between participants. Statistical comparison of results between groups was carried out after exclusion of these three participants and their matched controls, and the pattern of results did not change. Hence, no further distinction was made in the analysis. Passive attention was maintained through watching a silent closed-caption movie for the duration of each session. 


\subsection{EEG recording}

Participants were seated comfortably in a reclining chair with head support in a sound attenuating room. EEG was recorded using Synamps (Neuroscan, Inc., Acquire v4.3) from 30 sintered $\mathrm{Ag} / \mathrm{AgCl}$ electrodes arranged on an elastic cap in a standard 10-20 montage. There were four midline electrodes and 13 located over each hemisphere. A separate bi-polar channel was used to record vertical electro-oculographic (EOG) activity, with an electrode placed above and below the right eye. Data were recorded online with a sampling rate of $1-\mathrm{kHz}$, analog filtered from 0.05 to $200-\mathrm{Hz}$ ( $6 \mathrm{~dB} / \mathrm{Oct})$ and referenced to the $\mathrm{AFz}$ (midline) position. Electrode impedances (measured at $30-\mathrm{Hz}$ ) were below $3 \mathrm{k} \Omega$.

\subsection{Data processing}

Subsequent processing was carried out offline using Neuroscan Edit software (v4.3.1). Ocular artifact reduction was carried out using a regression analysis in combination with artifact averaging to correct for eye blinks (Semlitsch et al., 1986). The data were then low pass filtered (30-Hz, $24 \mathrm{~dB} /$ Octave), baseline corrected and epoched from $200 \mathrm{~ms}$ prior to the stimulus onset, to $600 \mathrm{~ms}$ post stimulus onset. Any epochs still containing activity exceeding $\pm 150 \mu \mathrm{V}$ were excluded from further analysis, with an average of 470 ( 1 s.d. = 36) epochs accepted in each block. Accepted epochs were averaged to reveal the N1 CAEP. Finally, the data were rereferenced to the average over the scalp electrodes.

\subsection{Analysis}

The analysis was carried out in two stages. In the first stage, the N1 evoked response was analyzed in the sensor space. The N1 response was objectively identified as the peak amplitude in the latency range of 70-130 ms post-stimulus onset using global field power (GFP). The mean amplitude, over a latency range $\pm 10 \mathrm{~ms}$ about this peak, was then obtained for comparison between groups at the vertex. In all cases where assumptions of normality of distribution and equality of variance were met, repeated-measures parametric tests were used to compare means between matched groups (Bland and Altman, 1994). Statistical comparison of the overall scalp field data was also carried out using non-parametric randomization tests (Koenig et al., 2011). In the second stage dipole source analysis was performed using Neuroscan Source software (v2.0). Modeling was performed on grand average waveforms from each condition and on the participant's mean data from each condition. A symmetrical 2-dipole model (one dipole in each hemisphere such that the dipole locations in the right hemisphere will have an equivalent position to the left hemisphere) was automatically applied using a classical 3-shell spherical head model. Source activity was allowed to vary in orientation and strength in a latency window of $\pm 10 \mathrm{~ms}$ around the $\mathrm{N} 1$ peak identified in the GFP so as to maximize the goodness-of-fit.

Mean group differences in dipole location and strength were assessed using repeated-measures ANOVA (Bland and Altman, 1994). Differences in mean dipole orientation were assessed using the Watson-Williams test (Zar, 1984).

\section{Results}

\subsection{Pure tone sensitivity thresholds}

Independent samples $t$-tests showed that there were no statistically significant differences in pure tone thresholds between UDs and MCs at either $0.5-\mathrm{kHz}\left(t_{34}=0.83, p=0.25\right)$ or $4-\mathrm{kHz}\left(t_{34}=0.89\right.$, $p=0.15)$. See Fig. 1 .

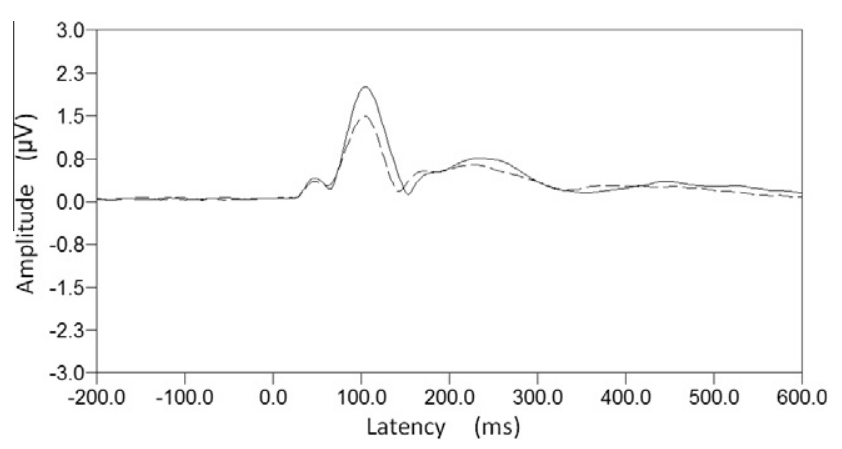

Fig. 2a. Grand mean GFP waveforms in the $0.5-\mathrm{kHz}$ condition for unilaterally deaf (solid lines) and matched control participants (dashed lines).

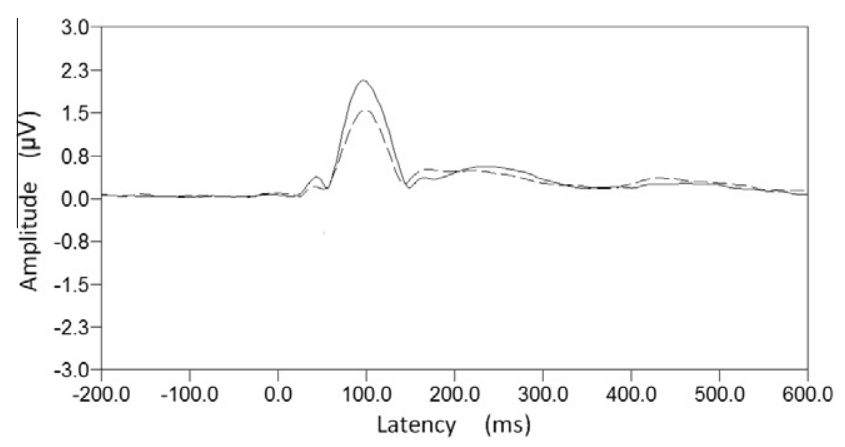

Fig. 2b. Grand mean GFP waveforms in the $4-\mathrm{kHz}$ condition for unilaterally deaf (solid lines) and matched control participants (dashed lines).

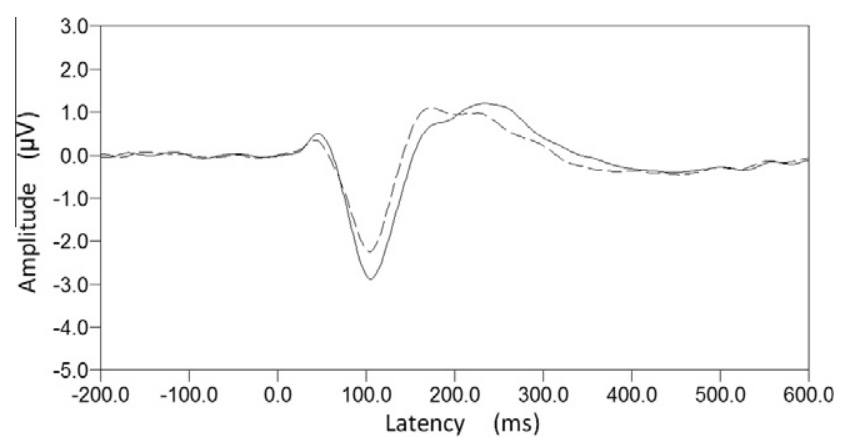

Fig. 2c. Grand mean ERP waveforms from the $0.5-\mathrm{kHz}$ condition from the vertex recording position for unilaterally deaf (solid lines) and matched control participants (dashed lines).

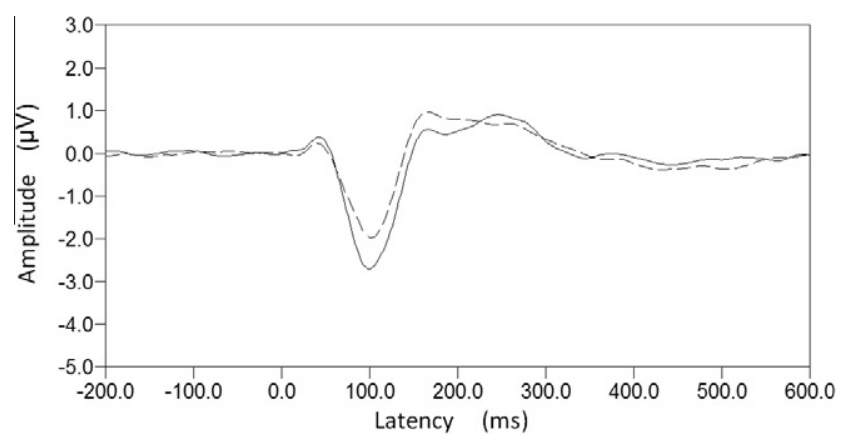

Fig. 2d. Grand mean ERP waveforms from the 4-kHz condition the vertex recording position for unilaterally deaf (solid lines) and matched control participants (dashed lines). 


\subsection{Analysis of CAEP waveforms and scalp fields}

Figs. 2a and 2b shows the grand mean GFP waveforms used for identifying the $\mathrm{N} 1$ peak for $0.5-\mathrm{kHz}$ and $4-\mathrm{kHz}$ stimulus conditions, respectively. Mean response amplitudes of $\mathrm{N} 1$ recorded from the vertex are displayed in Figs. $2 \mathrm{c}$ and $2 \mathrm{~d}$, for $0.5-\mathrm{kHz}$ and $4-\mathrm{kHz}$ stimulus conditions, respectively. There is a consistent trend for greater mean response amplitudes in UDs (solid line), compared with MCs (dashed line). In the $0.5-\mathrm{kHz}$ condition the mean amplitude was $2.86 \mu \mathrm{V}$ (s.d. $=1.00$ ) and $2.26 \mu \mathrm{V}$ (s.d. $=0.94$ ) for UDs and MCs, respectively. A paired-samples $t$-test revealed that these trends were not statistically significant $\left(t_{17}=1.76, p=0.09\right)$. In the 4$\mathrm{kHz}$ condition the mean amplitude was $2.67 \mu \mathrm{V}($ s.d. $=0.99)$ and $1.97 \mu \mathrm{V}$ (s.d. $=0.78$ ) for UDs and MCs, respectively. A pairedsamples $t$-test revealed that these mean differences were statistically significant $\left(t_{17}=2.57, p=0.02\right)$.

Fig. 3 displays the topographic maps corresponding to the grand mean waveforms from each stimulus condition. In MCs, the N1 response between each hemisphere is asymmetrical with the region of maximal N1 activity located over the contralateral hemisphere. In UDs, the response from each hemisphere appears more symmet- rical. These differences approached statistical significance in the $0.5-\mathrm{kHz}$ condition $(p=0.09)$ and were statistically significant in the $4-\mathrm{kHz}$ condition $(p=0.03)$.

\subsection{Dipole source analysis}

The goodness-of-fit for the grand average AEPs varied between $99.4 \%$ and $99.7 \%$ for both stimuli and both groups. Individual mean data for each participant achieved a goodness-of-fit between $94.4 \%$ and $99.7 \%$

The locations, orientations and strengths of the dipoles in each hemisphere quantify the activity reflected by the scalp topographies that are illustrated in Fig. 3. Table 1 summarizes the results of the grand mean dipole source analysis.

\subsubsection{Dipole location}

Fig. 4 displays the locations of dipoles from the grand average analysis in the three orthogonal planes (coronal, axial and sagittal). The dipole $x, y, z$ coordinates were measured in millimeters, where $x$ extends from left (negative values) to right (positive values) and 0 is on the midline. The $y$ axis extends anterior (positive values) to

\section{(a)}

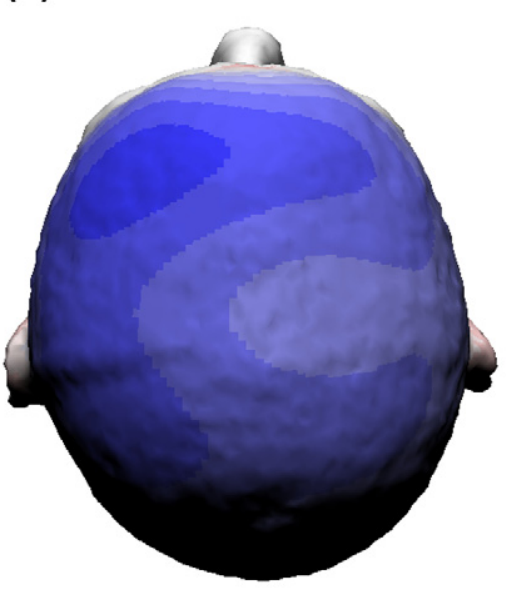

$\mathrm{MC}$

\section{(b)}

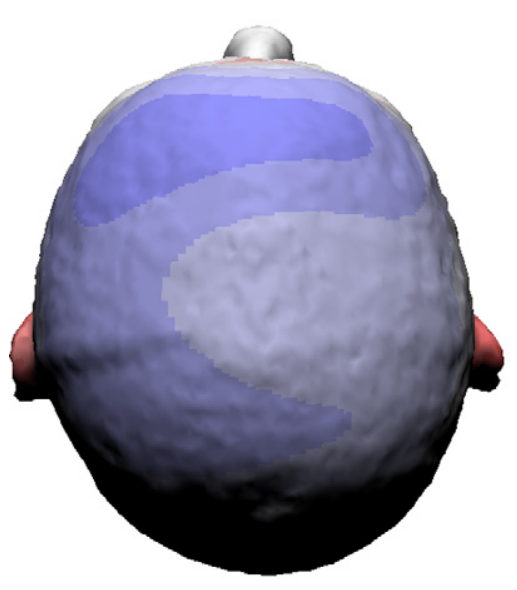

$\mathrm{MC}$
$0.5-\mathrm{kHz}$
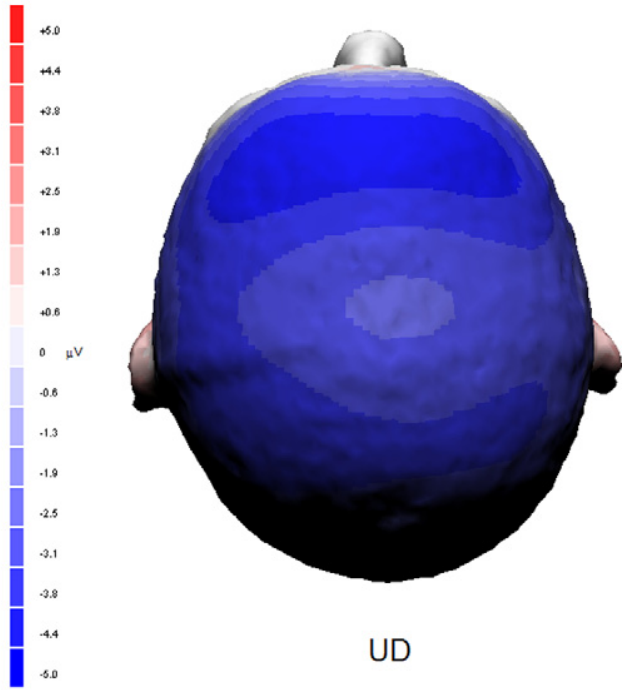

UD

$4-\mathrm{kHz}$

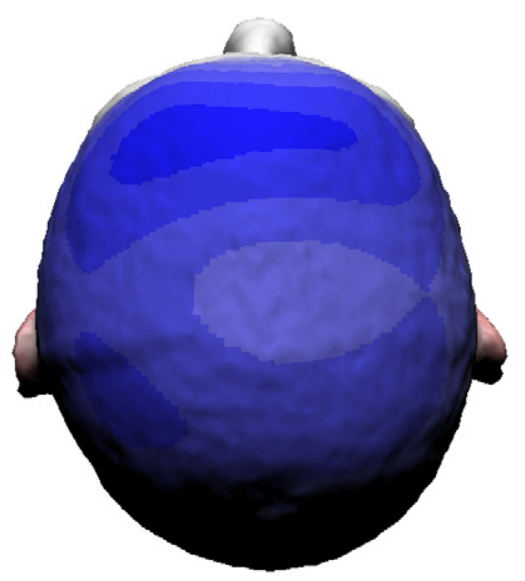

UD

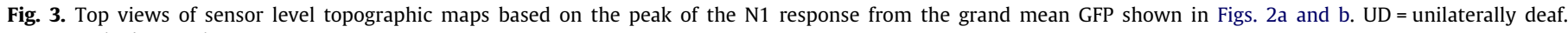
$\mathrm{MC}=$ matched control. 
Table 1

Summary of dipole source analysis for grand mean data.

\begin{tabular}{|c|c|c|c|c|c|c|c|c|c|c|c|c|c|c|}
\hline & \multicolumn{7}{|c|}{ Contralateral } & \multicolumn{7}{|c|}{ Ipsilateral } \\
\hline & \multicolumn{3}{|c|}{ Location (mm) } & \multicolumn{3}{|c|}{ Orientation $\left({ }^{\circ}\right)$} & \multirow[t]{2}{*}{ Strength (nAm) } & \multicolumn{3}{|c|}{ Location (mm) } & \multicolumn{3}{|c|}{ Orientation $\left(^{\circ}\right)$} & \multirow[t]{2}{*}{ Strength (nAm) } \\
\hline & $x$ & $y$ & $z$ & $z / x$ & $y / x$ & $z / y$ & & $x$ & $y$ & $z$ & $z / x$ & $y / x$ & $z / y$ & \\
\hline \multicolumn{15}{|c|}{$0.5-k H z$} \\
\hline UD & -39.5 & 8.4 & 38.7 & -100 & -110 & -64 & 40 & 45.8 & 8.4 & 38.7 & -84 & -79 & -62 & 29 \\
\hline MC & -39.9 & 7.7 & 46 & -91 & -93 & -64 & 30 & 46.2 & 7.7 & 46 & -91 & -91 & -61 & 18 \\
\hline \multicolumn{15}{|c|}{$4-k H z$} \\
\hline UD & -47.3 & 8.2 & 45.4 & -99 & -101 & -50 & 54 & 47.3 & 8.2 & 45.4 & -94 & -94 & -42 & 29 \\
\hline MC & -51.7 & 8.1 & 53.9 & -96 & -97 & -46 & 33 & 51.7 & 8.1 & 53.9 & -83 & -84 & -44 & 25 \\
\hline
\end{tabular}
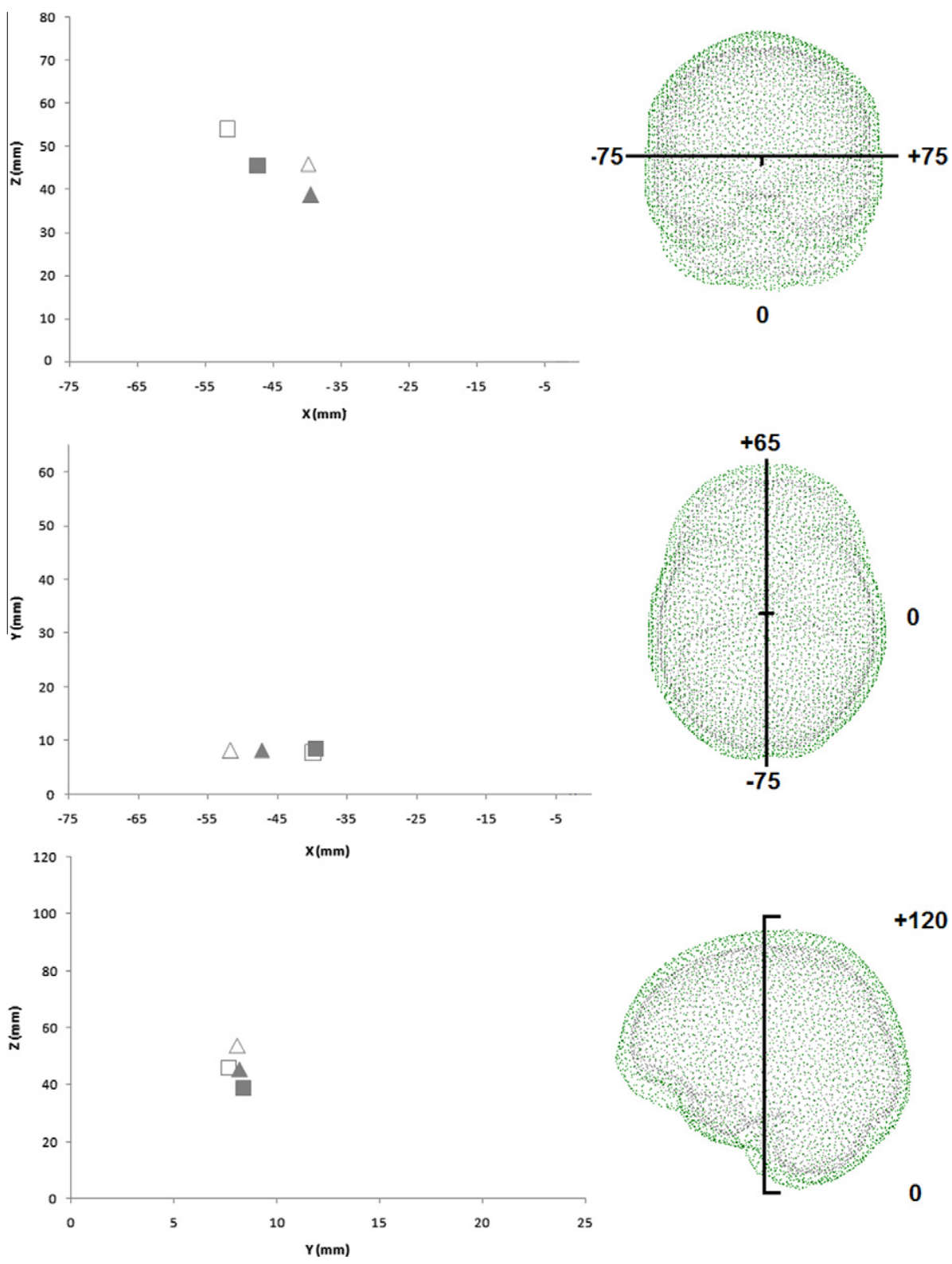

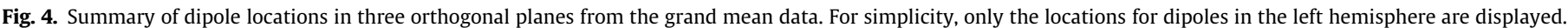

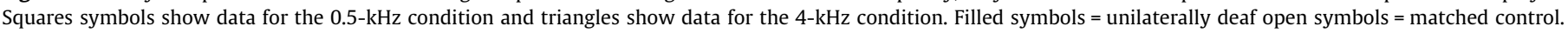

posterior (negative value) and 0 is on the midline. The $z$ axis extends superior (positive values) to inferior (positive values) and 0 is at the base of the brain in the same plane as the bottom of the cerebellum.

The top panel of Fig. 4 shows dipole locations in the coronal plane. The dipoles in the $4 \mathrm{kHz}$ condition showed a tendency towards a more medial location in UDs. However, there were no sta- tistically significant differences in either the $0.5-\mathrm{kHz}$ condition $\left(F_{(1,17)}=0.11 ; p=0.75\right)$ or $4-\mathrm{kHz}$ condition $\left(F_{(1,17)}=0.38 ; p=0.55\right)$.

The middle panel of Fig. 4 shows dipole locations in the axial plane. There were no statistically significant differences between groups in either the $0.5-\mathrm{kHz}$ condition $\left(F_{(1,17)}=0.002 ; p=0.97\right)$ or 4-kHz condition $\left(F_{(1,17)}=0.74 ; p=0.40\right)$. 


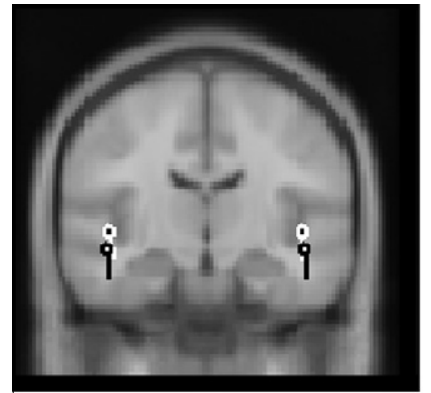

C

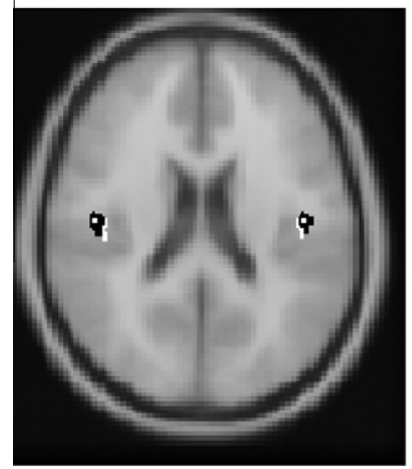

|

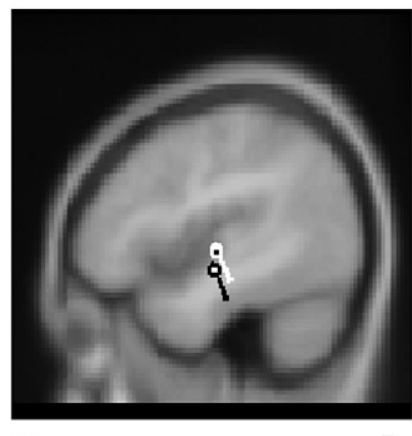

F
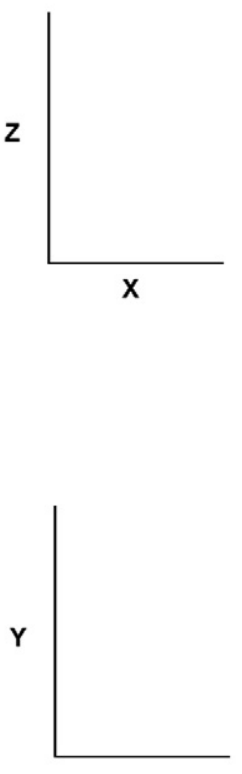

$\mathbf{X}$

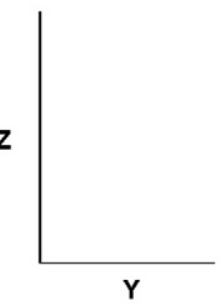

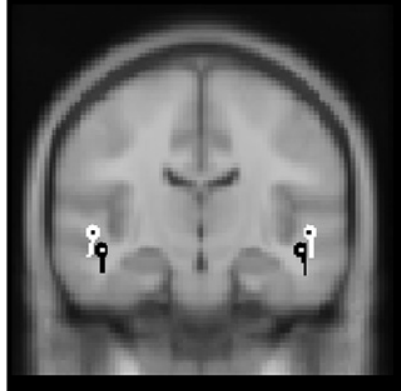

|

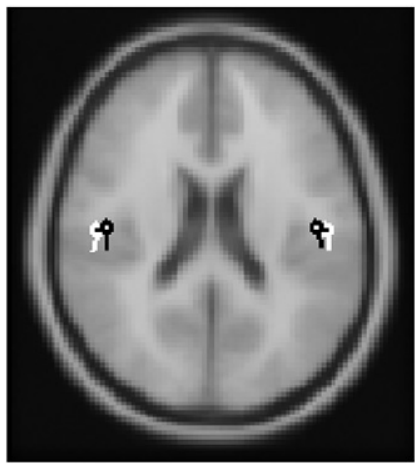

।

C

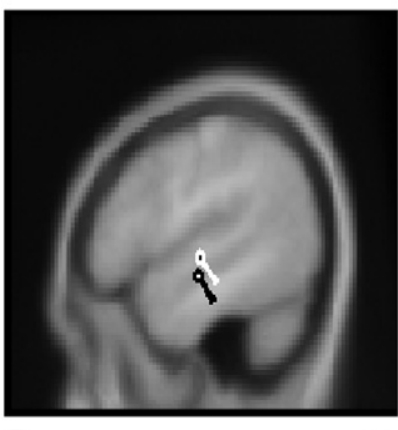

B

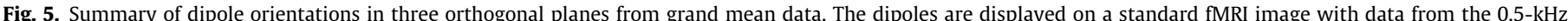

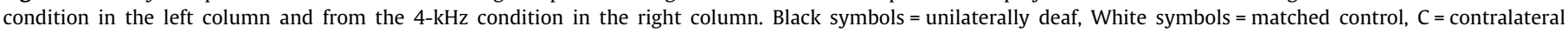
hemisphere, $\mathrm{I}=$ ipsilateral hemisphere, $\mathrm{F}=$ front, $\mathrm{B}=$ back.

The lower panel of Fig. 4 shows dipole locations in the sagittal plane. The dipoles show a trend towards more inferior locations in UD participants compared with MC participants. In the 0.5$\mathrm{kHz}$ condition the mean location in the $z$ axis was $39 \mathrm{~mm}$ $(\mathrm{SD}=9.5)$ for UDs and $48 \mathrm{~mm}(\mathrm{SD}=7.8)$ for MCs. In the $4 \mathrm{kHz}$ condition the mean location was $38 \mathrm{~mm}(\mathrm{SD}=9.8)$ in UDs and $47 \mathrm{~mm}$ ( $S D=8.9$ ) for MCs. A 2-factor (frequency x group) ANOVA revealed that this difference was not statistically significant in the $0.5-\mathrm{kHz}$ condition $\left(F_{(1,17)}=1.51 ; p=0.24\right)$ but it was significant in the 4$\mathrm{kHz}$ condition $\left(F_{(1,17)}=12.5 ; p=0.003\right)$.

\subsubsection{Orientation}

The orientation of the dipoles is described in terms of their angles with the coronal, axial and sagittal planes. An angle of $-90^{\circ}$ indicates vertical orientations in each plane. Angles between $0^{\circ}$ and $-90^{\circ}$ indicate orientations to the left in the coronal and axial planes, and more anterior in the sagittal plane. Angles between $-90^{\circ}$ and $-180^{\circ}$ indicate orientations to the right in coronal and axial planes, and more posterior in sagittal planes. Grand average dipole fits are displayed in Fig 5.
Fig. 5 (top panel) shows the orientations of dipoles in the coronal plane. The dipoles in the hemisphere contralateral to the ear of stimulation were generally orientated more medially for UD participants compared with MC participants. $\left(0.5-\mathrm{kHz}\right.$ : UD $=-93^{\circ}$ $(\mathrm{SD}=14), \quad \mathrm{MC}=-80^{\circ} \quad(\mathrm{SD}=16) ; 4-\mathrm{kHz}: \quad \mathrm{UD}=-104^{\circ} \quad(\mathrm{SD}=17)$, $\mathrm{MC}=-86^{\circ}(\mathrm{SD}=25)$; These differences were statistically significant in both the $0.5-\mathrm{kHz}$ condition $\left(F_{(1,17)}=6.65 ; p=0.01\right)$ and the 4$\mathrm{kHz}$ condition $\left(F_{(1,17)}=6.04 ; p=0.02\right)$. However, for the dipole in the ipsilateral hemispheres, neither difference reached statistical significance $\left(0.5-\mathrm{kHz}, F_{(1,17)}=3.11 ; p=0.09 ; 4-\mathrm{kHz}, F_{(1,17)}=1.16\right.$; $p=0.29$ ).

Fig. 5 (middle panel) shows the dipole orientations in the axial plane. Contralateral hemisphere dipoles in the $0.5-\mathrm{kHz}$ condition showed no statistically significant differences in orientation between participants and controls $\left(F_{(1,17)}=0.74 ; p=0.39\right)$. In the 4$\mathrm{kHz}$ condition the dipoles in the contralateral hemisphere of UD participants were orientated medially whereas those of MC participants were more tangentially orientated. This difference between groups was statistically significant $\left(F_{(1,17)}=4.59 ; p=0.04\right)$. The opposite pattern arose for dipole in the ipsilateral hemisphere 

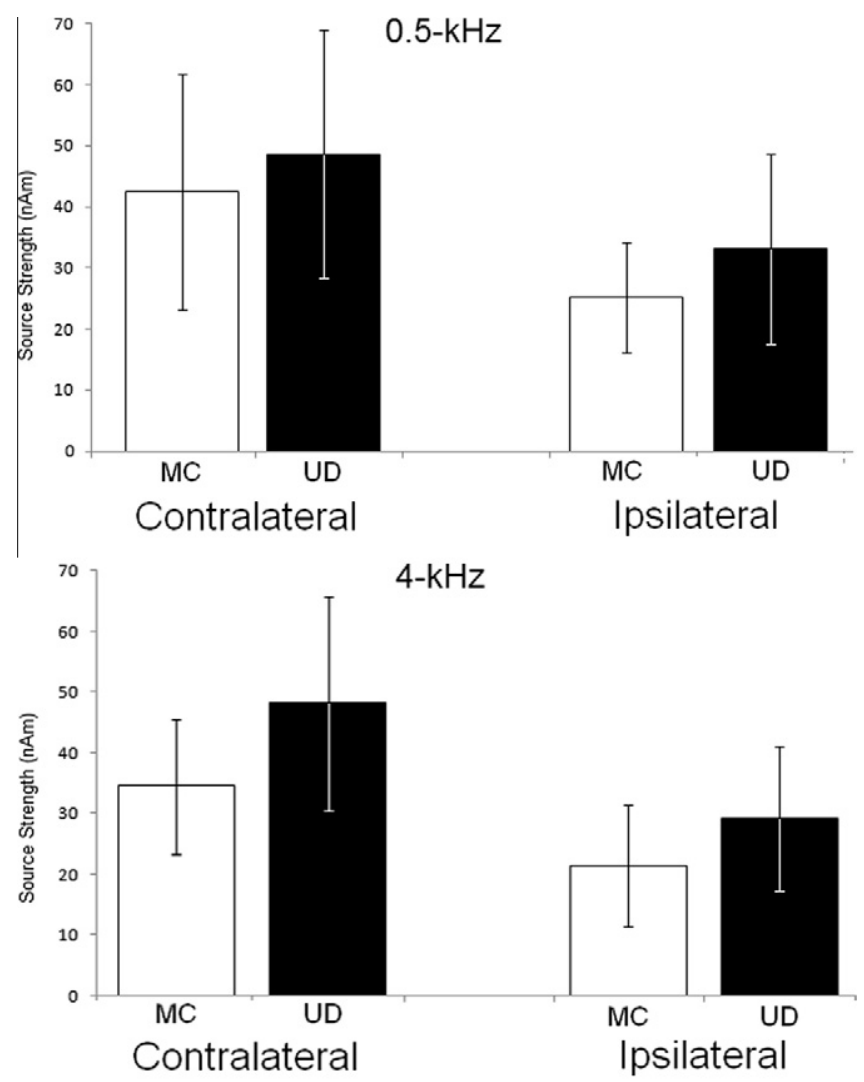

Fig. 6. Mean dipole source strengths. Error bars indicate \pm 1 s.d. UD $=$ unilaterally deaf. $\mathrm{MC}=$ matched control.

although there were no statistically significant differences for either stimulus condition $\left(0.5-\mathrm{kHz}, F_{(1,17)}=2.85 ; p=0.10 ; 4-\mathrm{kHz}\right.$, $\left.F_{(1,17)}=1.09 ; p=0.30\right)$.

Fig. 5 (lower panel) shows the dipole orientations in the sagittal plane. Group comparisons of the dipole orientations in this plane showed no statistically significant differences in the contralateral hemisphere for the $0.5-\mathrm{kHz}\left(F_{(1,17)}=0.50 ; p=0.48\right)$. However, contralateral hemisphere dipoles in unilaterally deaf participants were orientated significantly more anteriorly in the $4-\mathrm{kHz}$ condition $\left(F_{(1,17)}=5.10 ; p=0.03\right)$. There were no statistically significant differences for orientations in the ipsilateral hemispheres $(0.5-\mathrm{kHz}$, $\left.F_{(1,17)}=0.04 ; p=0.84 ; 4-\mathrm{kHz}, F_{(1,17)}=0.77 ; p=0.39\right)$.

\subsubsection{Source strengths}

Fig. 6 shows the source strengths for dipoles in the contralateral and ipsilateral hemispheres in each stimulus condition. The trends towards greater response amplitudes in individuals with unilateral deafness are reflected by higher dipole strengths in both hemispheres compared with matched controls. Consequently the hemispheric lateralization in dipole strengths in each condition is preserved following unilateral deafness i.e. the contralateral dipole remains stronger than the ipsilateral dipole. For the $0.5-\mathrm{kHz}$ stimulus, the dipoles are around 9\% stronger and for the $4-\mathrm{kHz}$ stimulus the dipoles are around $16 \%$ stronger in participants with unilateral deafness. Separate within-subjects ANOVAs were performed for each stimulus condition (hemisphere [2] $\times$ group [2]). In the 0.5$\mathrm{kHz}$ condition a statistically significant main effect of hemisphere was observed, with a stronger response from the contralateral hemisphere $\left(F_{(1,17)}=20.32 ; p<0.001\right)$. However, the trend for higher dipole strengths was not statistically significant $\left(F_{(1,17)}=1.73\right.$; $p=0.21)$, and there was no interaction $\left(F_{(1,17)}=0.05 ; p=0.83\right)$. In the 4-kHz condition a statistically significant main effect of hemisphere was again observed $\left(F_{(1,17)}=23.02 ; p<0.001\right)$. The trend for greater dipole strengths in individuals with unilateral deafness was also statistically significant $\left(F_{(1,17)}=7.09 ; p=0.02\right)$. No interaction effects were found, demonstrating that the trend for increased amplitudes in those with unilateral deafness was apparent in both hemispheres $\left(F_{(1,17)}=1.14 ; p=0.19\right)$.

\section{Discussion}

This study had the aim of comparing the scalp field and source localization data of the N1 CAEP between a group of adult humans with late onset unilateral profound deafness and a control group. The results show that there is a difference in cortical auditory evoked potentials between the groups when tested under identical, monaural listening conditions. Based on scalp field data, these differences include increased N1 amplitudes and reduced hemispheric asymmetries in the individuals with unilateral deafness. This is consistent with experience-related plasticity within the adult CAS. The study therefore corroborates the only previous evidence for such plasticity measured via AEPs (Ponton et al., 2001). The results also extend the previous findings in individuals with unilateral deafness by demonstrating that these changes may be reflected by increased dipole strengths bilaterally, and altered dipole locations and orientations. This finding is important as it may help explain the inconsistent findings in previous studies (Fujiki et al., 1998; Hine et al., 2008; Vasama et al., 1995, 2001) as well as providing evidence that dipole source analysis may be a reliable tool for characterizing changes in hemispheric asymmetries. Such information is important as this provides a robust means of studying plasticity that might arise in the adult human CAS following injury-induced sensory deprivation, auditory training or rehabilitation strategies.

\subsection{Scalp field data}

A trend for greater $\mathrm{N} 1$ amplitudes and reduced hemispheric asymmetries was observed in individuals with unilateral deafness compared with matched controls in both stimulus conditions. A similar pattern was also observed irrespective of the ear of deafness. An increase in activity, as measured by auditory evoked potentials, has been reported previously in association with other forms of experience-related plasticity following partial auditory deprivation. For example, Salvi et al. (2000) described increased amplitude of activity in the inferior colliculus of animal models with high frequency hearing loss at frequencies below the region of the hearing loss. Dietrich et al. (2001) reported a similar increase in the mean $\mathrm{N} 1 \mathrm{~m}$ amplitude to stimulation at the edge frequency in adult humans with steeply sloping high frequency hearing losses compared to other, much lower frequencies and Ponton et al. (2001) described an increase in N1 amplitude over the ipsilateral hemisphere relative to the intact ear in adults with profound unilateral deafness. Two phenomena could explain an increase in amplitude in response to stimulation of the intact ear; an increase in the number of responsive neurons and/or an increase in the synchrony of neural firing. Based on animal experiments investigating abrupt unilateral deafness, binaurally sensitive neurons that were primarily responsive to the deafened ear prior to the onset of deafness have been found to become more responsive to the intact ear after the onset (McAlpine et al., 1997). Stimulation of the intact ear might therefore cause more neurons to respond than previously. With respect to synchrony of firing, previous studies suggest that this form of plasticity will be most apparent in the hemisphere ipsilateral to the intact ear (Scheffler et al., 1998; Ponton et al., 2001). Any enhancement of neural activity to stimulation of the 
intact ear will result in an increase in the synchrony of firing between neural populations in the contralateral and ipsilateral hemispheres.

Whilst a trend for this effect was apparent in both stimulus conditions, a statistically significant difference was only observed in the $4-\mathrm{kHz}$ condition. Post-hoc power analysis revealed a standardized effect size of 0.62 in the $0.5-\mathrm{kHz}$ condition and 0.77 in the 4$\mathrm{kHz}$ condition. This suggests that a similar pattern of changes occurred to both low and high frequency stimuli, but that the lack of a statistically significant effect for the lower frequency stimulus was due to the experiment having insufficient statistical power. The reason for the smaller effect size at $0.5-\mathrm{kHz}$ is unclear but could be related to frequency specific differences in the underlying component structure of the N1 CAEP and subsequent differences in degrees of hemispheric lateralization (Verkindt et al., 1995; Pantev et al., 1998; Picton et al., 1999). In addition, previous studies have suggested frequency-specific differences in the cortical representation of binaural interactions (e.g. Khosla et al., 2003).

A further consideration to note is the differences in mean pure tone hearing thresholds at $0.5-\mathrm{kHz}$ and $4-\mathrm{kHz}$. Mean thresholds were higher in both groups by approximately $10 \mathrm{~dB}$ for the 4$\mathrm{kHz}$ stimulus. This is likely to be age-related hearing loss, a feature of which is abnormal growth of loudness. However, abnormal loudness growth is unlikely to explain the greater amplitudes in participants with unilateral deafness for several reasons: (1) the intact ear of participants with unilateral deafness was matched for pure tone sensitivity to the control participants, (2) a similar pattern of results was observed for low frequency stimuli where there was little or no hearing loss, and (3) the amplitude differences between the groups were also accompanied by differences in scalp topographies.

\subsection{Dipole source analysis}

It is known that the N1 CAEP is composed of several sources in each hemisphere (Naatanen and Picton, 1987). However, the aim of the present dipole source analysis was not to characterize all components in each hemisphere, but rather to represent at least a first approximation and centre of gravity of these sources such that differences between groups might be used to characterize plasticity. Furthermore, since the different scalp field topographies from each group must be caused by at least partially different configurations of these sources within the CAS (Michel et al., 2004), then the pattern of changes in the dipoles should provide an indication of the pattern of changes within the CAS.

In the group with unilateral deafness, the dipole strengths tended to be greater in both the ipsilateral and contralateral hemispheres compared with the control group. The dipole orientation tended to shift towards the midline, where the activity was maximal, and the dipoles were located more inferiorly. This final difference is consistent with previous findings (Khosla et al., 2003). The most plausible explanation for this pattern of findings is an increase in the strength of activity from within the hemisphere ipsilateral to the ear of stimulation. For instance, if the source activity increased similarly in both contralateral and ipsilateral hemispheres as the bilateral change in dipole strengths might suggest, then no shift in the scalp field topographies would have occurred. However, no shift in dipole orientation alone could explain the change in scalp topography. If such a shift really did occur then no net change in amplitude would occur and the increase in dipole strength would not have been recorded.

The findings of this study suggest that acquired sensory deprivation in adults leads to a change in the neural population generating the N1 response. Similar changes in dipole parameters have already been reported elsewhere when hemispheric asymmetries are apparent following the processing of changes to low and high frequency stimuli (e.g. Dimitrijevic et al., 2008). The interpretation of the findings in the present study (i.e. increased ipsilateral hemisphere activity) is consistent with previous reports following idiopathic unilateral sensorineural hearing loss (e.g. Morita et al., 2007). However, in this report a dramatic increase in ipsilateral dipole strengths was reported immediately following onset of hearing loss. The discrepancy between these and the present results may be explained by the use of a fixed dipole model (i.e. the location and orientation of the dipoles being set apriori) rather than a rotating dipole used here. Although source waveforms are relatively robust against moderate inaccuracies in location, they are vulnerable to changes in orientation (Lutkenhoner and Mosher, 2007). Hence a fixed dipole may not be appropriate for reflecting at least partially overlapping fields of N1 sources that are encountered in EEG; otherwise, any change in scalp field data such as those reported presently may only be reflected by a considerable increase in the ipsilateral dipole strength.

\section{Conclusions}

The present results revealed evidence of experience-related plasticity in unilaterally deaf adult humans. This is important because a number of previous studies have not done so, in particular where dipole modeling has been used to quantify the activity arising in each hemisphere. It is apparent that AEPs are sensitive to experience-related plasticity due to profound unilateral deafness and the mixed findings reported previously are probably due, at least in part, to limitations in methodology. Future studies should account for altered dipole location, orientation and strength when quantifying AEP changes following experience-related plasticity. In addition, the present findings clarify and extend previous work by demonstrating a similar pattern of changes irrespective of ear of deafness or frequency of stimulation. One explanation for these results is an increased responsiveness of neurons to stimulation of the intact ear as a result of experience-related plasticity. Further studies are needed to characterize the extent and time course of experience-related plasticity as this may help to elucidate the physiological mechanisms involved, and clarify any perceptual consequences.

\section{Acknowledgement}

This work was funded by a studentship from the Medical Research Council, UK.

\section{References}

Bilecen D, Seifritz E, Radu EW, Schmid N, Wetzel S, Probst R, et al. Cortical reorganization after acute unilateral hearing loss traced by fMRI. Neurology 2000;54:765-7.

Bland JM, Altman DG. Matching. Brit Med J 1994;309:1128.

Dietrich V, Nieschalk M, Stoll W, Rajan R, Pantev C. Cortical reorganization in patients with high frequency cochlear hearing loss. Hear Res 2001;158:95-101.

Dimitrijevic A, Michalewski HJ, Zeng FG, Pratt H, Starr A. Frequency changes in a continuous tone: auditory evoked potentials. Clin Neurophysiol 2008;119: 2111-24.

Firszt JB, Ulmer JL, Gaggl W. Differential representation of speech sounds in the human cerebral hemispheres. Anat Rec Part A 2006;288:345-57.

Fujiki N, Naito Y, Nagamine T, Shiomi Y, Hirano S, Honjo I, et al. Influence of unilateral deafness on auditory evoked magnetic field. Neuroreport 1998;9: 3129-33.

Hanss J, Veuillet E, Adjout K, Besle J, Collet L, Thai-Van H. The effect of long-term unilateral deafness on the activation pattern in the auditory cortices of Frenchnative speakers: influence of deafness side. BMC Neurosci 2009;10:23.

Hine J, Debener S. Late auditory evoked potentials asymmetry revisited. Clin Neurophysiol 2007;118:1274-85.

Hine J, Thornton R, Davis A, Debener S. Does long-term unilateral deafness change auditory evoked potential asymmetries? Clin Neurophysiol 2008;119:576-86.

Khosla D, Ponton CW, Eggermont JJ, Kwong B, Don M, Vasama J-P. Differential ear effects of profound unilateral deafness on the adult human central auditory system. J Assoc Res Otolaryngol 2003;4:235-49. 
Koenig T, Kottlow M, Stein M, Melie-Garcia L. Ragu: a free tool for the analysis of EEG and MEG event-related scalp field data using global randomization statistics. Comput Intell Neurosci 2011;2011:938925.

Langers DR, van Dijk P, Backes WH. Lateralization, connectivity and plasticity in the human central auditory system. Neuroimage 2005;28:490-9.

Lutkenhoner B, Mosher JC. Source analysis of auditory evoked potentials and fields. In: Burkard RF, Don M, Eggermont JJ, editors. Auditory evoked potentials. Basic principles and clinical applications. Baltimore: Lippincott, Williams and Wilkins; 2007. p. 546-69.

McAlpine D, Martin RL, Mossop JE, Moore DR. Response properties of neurons in the inferior colliculus of the monaurally deafened ferret to acoustic stimulation of the intact ear. J Neurophysiol 1997;78:767-79.

Michel CM, Murray MM, Lantz G, Gonzalez S, Spinelli L, Grave de Peralta R. EEG source imaging. Clin Neurophysiol 2004;115:2195-222.

Morita T, Hiraumi H, Fujiki N, Naito Y, Nagamine T, Fukuyama H, et al. A recovery from enhancement of activation in auditory cortex of patients with idiopathic sudden sensorineural hearing loss. Neurosci Res 2007;58:6-11.

Naatanen R, Picton T. The N1 wave of the human electric and magnetic response to sound: a review and an analysis of the component structure. Psychophysiology 1987;24:375-425.

Neuman AC. Central auditory system plasticity and aural rehabilitation of adults. J Rehabil Res Dev 2005;42:169-86.

Oldfield RC. The assessment and analysis of handedness: the Edinburgh inventory. Neuropsychologia 1971;9:97-113.

Pantev C, Ross B, Berg P, Elbert T, Rockstroh B. Study of the human auditory cortices using a whole-head magnetometer: left vs. right hemisphere and ipsilateral vs. contralateral stimulation. Audiol Neurootol 1998;3:183-90.

Picton TW, Alain C, Woods DL, John MS, Scherg M, Valdes-Sosa P, et al. Intracerebral sources of human auditory-evoked potentials. Audiol Neurootol 1999;4: 64-79.
Ponton CW, Vasama JP, Tremblay K, Khosla D, Kwong B, Don M. Plasticity in the adult human central auditory system: evidence from late-onset profound unilateral deafness. Hear Res 2001;154:32-44.

Popelar J, Erre JP, Aran JM, Cazals Y. Plastic changes in ipsi-contralateral differences of auditory cortex and inferior colliculus evoked potentials after injury to one ear in the adult guinea pig. Hear Res 1994;72:125-34.

Salvi RJ, Wang J, Ding D. Auditory plasticity and hyperactivity following cochlear damage. Hear Res 2000;147:261-74.

Scheffler K, Bilecen D, Schmid N, Tschopp K, Seelig J. Auditory cortical responses in hearing subjects and unilateral deaf patients as detected by functional magnetic resonance imaging. Cereb Cortex 1998;8:156-63.

Semlitsch HV, Anderer P, Schuster P, Presslich O. A solution for reliable and valid reduction of ocular artifacts, applied to the P300 ERP. Psychophysiology 1986;23:695-703.

Vasama JP, Makela JP, Pyykko I, Hari R. Abrupt unilateral deafness modifies function of human auditory pathways. Neuroreport 1995;6:961-4.

Vasama JP, Marttila T, Lahin T, Makela JP. Auditory pathway function after vestibular schwannoma surgery. Acta Oto-Laryngol 2001;121:378-83.

Verkindt C, Bertrand O, Perrin F, Echallier JF, Pernier J. Tonotopic organization of the human auditory cortex: N100 topography and multiple dipole model analysis. Electroencephalogr Clin Neurophysiol 1995;96:143-56.

Woldorff MG, Tempelmann C, Fell J, Tegeler C, Gaschler-Markefski B, Hinrichs H, et al. Lateralized auditory spatial perception and the contralaterality of cortical processing as studied with functional magnetic resonance imaging and magnetoencephalography. Hum Brain Mapp 1999;7:49-66.

Zar JH. Biostatistical analysis. 2nd ed. London: Prentice-Hall International; 1984 